\title{
Light-ion production and fission studies using the MEDLEY facility at TSL
}

\author{
S. Pomp ${ }^{\star, 1}$, J. Blomgren ${ }^{1}$, M. Hayashi ${ }^{1,2}$, P. Mermod ${ }^{1}$, A. Öhrn ${ }^{1}$, N. Olsson ${ }^{1}$, \\ M. Österlund ${ }^{1}$, A. Prokofiev ${ }^{3}$, U. Tippawan ${ }^{4}$ \\ ${ }^{1}$ Department of Neutron Research, Uppsala University, Sweden \\ ${ }^{2}$ Department of Advanced Energy Engineering Science, Kyushu University, Japan \\ ${ }^{3}$ The Svedberg Laboratory, Uppsala University, Sweden \\ ${ }^{4}$ Fast Neutron Research Facility, Chiang Mai University, Thailand \\ E-mail: Stephan.Pomp@tsl.uu.se
}

\begin{abstract}
The MEDLEY facility at the The Svedberg Laboratory (TSL) in Uppsala has successfully been used in several light-ion production and elastic scattering experiments at $96 \mathrm{MeV}$. The recent upgrade of the neutron beam facility at TSL now makes the energy range up to $175 \mathrm{MeV}$ accessible. To match the higher energies MEDLEY has been equipped with larger CsI detectors and first tests runs have been performed. The research program at MEDLEY has been extended to include, e.g., studies of neutron-induced fission, especially angular distributions of the fission fragments, over a wide energy range. We present the current status of the facility and the planned research program.
\end{abstract}

International Workshop on Fast Neutron Detectors University of Cape Town, South Africa

April 3 - 6, 2006

\footnotetext{
${ }^{*}$ Speaker
} 


\section{Introduction}

Understanding how neutrons interact with nuclei is a difficult task since it involves interactions of a large number of nucleons with each other. This is especially true for production of complex particles $(A \geq 2)$ by neutrons in the energy range of 20-200 MeV. Nevertheless, a wide variety of different applications involving interactions of fast neutrons with nuclei have developed rapidly during the past years. Examples are dosimetry at commercial aircraft altitudes and in space and radiation treatment of cancer within the field of medicine, soft-error effects in computer memory within electronics, and energy production and transmutation of nuclear waste within energy applications. Common for all these applications is the need for high-quality nuclear data for specific nuclei over a wide energy range on the one hand, and the development of reliable nuclear model codes to extrapolate into unmeasured areas on the other hand.

The MEDLEY facility, located at the The Svedberg Laboratory (TSL) in Uppsala, Sweden, has over the past years performed measurements of double-differential cross sections for the production of light ions by $96 \mathrm{MeV}$ neutrons [1-4]. Recently, we have started a program on measuring angular distributions of fission fragments [5]. The facility has also proven to be a valuable tool in the search for three-body force effects [6-7]. All these measurements have been performed at the "old" neutron beam at TSL [8]. At this beam, the neutron fluence above 100 $\mathrm{MeV}$, where the cyclotron has to operate in FM mode, becomes too low to collect good statistics within reasonable time and it was therefore decided to construct a new neutron beamline with shorter distance from the neutron production point to the experimental area, thus delivering higher neutron fluxes. This new beamline is in operation since 2004 [9-10] and opens up the possiblity to extend the experimental program and measure neutron-induced reactions at energies up to $175 \mathrm{MeV}$.

\section{Light-ion production studies with MEDLEY}

\subsection{Medical applications}

It has been established that, due to cosmic-ray neutrons, airflight personnel receive among the largest radiation doses in civil work [11]. Even at space flight altitudes, neutrons give a large contribution to the radiation effects on both human tissue and electronic equipment [12]. Cancer treatment with fast neutrons is performed routinely at several facilities around the world, and represents today one of the largest therapy modalities besides the conventional treatments with photons and electrons. See, e.g., Ref. [13] for a review of this field.

The interaction of neutrons with tissue is very complex, and to a large extent unknown. Neutrons themselves make no damage, but if they induce a nuclear reaction, the emitted charged products interact with the surrounding matter. This interaction between secondary products and tissue is rather well known, but the cross sections for their production are most often poorly known, or even unknown. Due to the absence of high-quality data on neutron-induced nuclear reactions, the concept of kerma (Kinetic Energy Released in MAtter), integrating over a wide 
variety of radiation effects, has been used. With microscopic cross section data at hand, the kerma-based dose estimations become obsolete which could revolutionise dosimetry for fast neutrons [14].

In the energy range around $100 \mathrm{MeV}$, about half the dose delivered to human tissue by neutrons comes from elastic $n p$ scattering, another $10 \%$ from elastic neutron scattering of other nuclei than hydrogen, and the remaining $40 \%$ from neutron-induced emission of charged particles. Double-differential cross sections for the production of these charged particles have been and will be measured in tissue relevant material. Carbon data obtained with MEDLEY at $96 \mathrm{MeV}$ at the "old" neutron beam are still under analysis. A paper on double-differential cross sections for light-ion production by $96 \mathrm{MeV}$ neutrons on oxygen has been published recently [3]. Carbon data at $175 \mathrm{MeV}$ have been collected during 50 hours of beamtime in March this year. More data on carbon will be taken in May. It is planned to also collect oxygen data together with silicon data using $\mathrm{SiO}_{2}$ and $\mathrm{Si}$ targets.

\subsection{Neutron-induced electronic failures}

Cosmic radiation effects in aircraft electronics are well known since more than 20 years. When an electronic circuit is exposed to a charged particle, the latter can, by ionisation inside the sensitive volume, release enough charge to induce a flip in the memory content of a bit. This non-destructive effect is called a single-event upset (SEU). However, a detailed understanding is still lacking (for an overview, see, e.g., Ref. [15] and references therein).

At flight altitudes, as well as at sea level, neutrons and muons dominate the cosmic ray flux. Muons do not interact strongly with nuclei, and therefore neutrons are most important for SEU. Even onboard spacecraft, neutrons produced in the aluminum structure, contribute, on a level comparable to protons, to radiation effects in both electronics and human tissue [12].

Since neutrons have no charge, they can only interact via nuclear reactions, in which charged particles or a heavy recoil are created, that occasionally induce an SEU. Thus, similar to the case of medical applications, detailed knowledge of the nuclear interaction of neutrons with silicon, and even oxygen, are needed. These, together with an adequate description of the electrical and geometrical properties of the decives might lead to a full understanding of the SEU problem [16].

Cross section data on light-ion production in silicon induced by $96 \mathrm{MeV}$ neutrons have already been measured with MEDLEY [2]. SEU cross section data obtained by direct in-beam component testing some years ago $[17,18]$ seemed to indicate that the SEU cross section saturates at a neutron energy of about $100 \mathrm{MeV}$. However, recent measurements performed at TSL suggest that, for the most recent devices, the SEU cross section might reach a maximum value at a neutron energy of a few tens of $\mathrm{MeV}$ and then decrease again with energy [19]. This is supported by a recent study with the nuclear model code TALYS [20]. Since this issue is currently under some debate, there is a further reason to perform a high-quality measurement of double-differential cross sections of neutron-induced light-ion production in silicon and oxygen at $175 \mathrm{MeV}$. 


\subsection{Applications in transmutation technologies}

The interest in transmutation technologies, especially accelerator-driven systems (ADS), is rapidly growing. They involve neutrons at considerably higher energies than what has been utilised in present reactor applications.

Up to now, extensive data bases exist up to about $20 \mathrm{MeV}$, which is the relevant energy interval for conventional nuclear power and fusion research. With the proposed technologies, neutron energies up to $2 \mathrm{GeV}$ could become of interest. All ADS proposals deal with spallationtype neutron production methods, which give neutron spectra with an intensity distribution roughly like $1 / E_{n}$. The small number of neutrons at really high energies makes such data not being as important as mid-range data. Above, say, $200 \mathrm{MeV}$, direct reaction models assuming a single interaction (impulse approximation) works reasonably well, while at lower energies nuclear distortion plays a non-trivial role. This makes the $20-200 \mathrm{MeV}$ region the most important for new data [21].

Very little high-quality neutron-induced data exist in this domain. MEDLEY has already served as facility for the measurement of neutron-induced light-ion production on $\mathrm{Fe}, \mathrm{Pb}$ and $\mathrm{U}$ at $96 \mathrm{MeV}$ [4]. Above $100 \mathrm{MeV}$ there are virtually no neutron data that can be used to validate nuclear reaction model codes such as TALYS. From a technical point of few, lead and bismuth (cooling), iron (structure material) and the actinides to be transmuted (uranium, thorium, plutonium, etc.) are the most important. Therefore, we plan measurement of neutron-induced light-ion production on iron, lead, bismuth and uranium at $175 \mathrm{MeV}$.

\section{Fission studies with MEDLEY}

Neutron-induced fission reactions of ${ }^{235} \mathrm{U},{ }^{238} \mathrm{U}$ and ${ }^{209} \mathrm{Bi}$ are internationally recommended standards for monitoring high-energy neutron beams [22]. Fission is also one of the most important processes occuring in the spallation target and the reactor core of an ADS. Furthermore, data on high-energy $(n, f)$ are important for, e.g., developing the theoretical understanding of dynamic effects in the fission process.

Despite the importance of high-energy $(\mathrm{n}, \mathrm{f})$ data, few attempts have been made to measure fission cross sections on an absolute scale, i.e., versus the np scattering cross section, which is adopted as the primary neutron standard [22].

The current standard ${ }^{235} \mathrm{U}(\mathrm{n}, \mathrm{f})$ and ${ }^{238} \mathrm{U}(\mathrm{n}, \mathrm{f})$ cross sections recommended by IAEA [22] are based on the data sets of Lisowski et al. [23]. These data have been revised several times and a thorough description of the experimental technique is still awaited.

We plan to measure the ${ }^{238} \mathrm{U}(\mathrm{n}, \mathrm{f})$ cross section and angular distributions of fission fragments in the energy range of 20 to $175 \mathrm{MeV}$. First results at $20.9 \mathrm{MeV}$ have been published [5]. The strength of the employed experimental technique is the simultaneous measurement of fission fragments and $\mathrm{np}$ scattering events in the MEDLEY telescopes. Counting $\alpha$ particles from radioactive decay of the target nuclei gives the effective solid angle. 
This can be done either during the beamtime or without beam. Measuring angular distributions thus reduces to counting fragments and $\alpha$-particles.

\section{Experimental considerations}

Using MEDLEY at the new Uppsala neutron beam, we plan to measure double-differential cross sections for light-ion production on carbon, oxygen, silicon, iron, lead, bismuth and uranium at $175 \mathrm{MeV}$. Furthermore we will measure the ${ }^{238} \mathrm{U}(\mathrm{n}, \mathrm{f})$ cross section, together with angular distributions of the fission fragments, over the energy region of 20 to $175 \mathrm{MeV}$. The proposed target nuclei are of highest interest within the applications listed above, and, in addition, of key interest for model development.

\subsection{The MEDLEY facility}

The charged particles are detected by the MEDLEY setup [2]. It consists of eight threeelement telescopes mounted inside a $90 \mathrm{~cm}$ diameter evacuated reaction chamber. Each telescope has two fully depleted $\Delta \mathrm{E}$ silicon surface barrier detectors (the first is $50-60 \mu \mathrm{m}$ thick, the second 400-550 $\mu \mathrm{m}$ ) and one E CsI(Tl) detector. The time-of-flight (TOF) of the neutron and the charged particle triggering the event, obtained from the cyclotron RF and the timing signal from each telescope, is measured for each charged-particle event.

MEDLEY has been equipped with larger CsI detectors to be able to stop protons up to 180 $\mathrm{MeV}$. These new detectors have now been used during several runs and perform according to expectations. The CsI crystals have a total length of $100 \mathrm{~mm}$. The first $70 \mathrm{~mm}$ is made cylindrical with a diameter of $50 \mathrm{~mm}$ and the remaining $30 \mathrm{~mm}$ is tapered to $18 \mathrm{~mm}$ diameter to match the size of the readout system. The readout is performed by Hamamatsu S3204-08 photodiodes (PD). The crystals, toghether with the PD, are mounted inside an aluminum tube and have been manufactured by Saint-Gobain, France.

\subsection{Background}

During the first runs we have found a rather large background probably due to neutrons from the production target penetrating the concrete shielding. While this is no problem for other experiments, it could be for MEDLEY since each individual CsI detector consists of three orders of magnitude more "target" material than the actual target. For acceptance reasons, the registered background is small at the most forward angles but gets large at more backward angles. We anticipate, however, that the particle identification procedure and TOF windows in the offline analysis will filter out most of this background.

\subsection{Data-taking and analysis}

As mentioned above, we have started to collect data on ${ }^{12} \mathrm{C}(\mathrm{n}, \mathrm{lcp})$ in March this year. We will improve the statistics by further runs in May. These data will be analysed by one of us 
$(\mathrm{MH})$ at Kyushu University in Japan. For the light-ion production studies from $\mathrm{Fe}, \mathrm{Pb}, \mathrm{Bi}$ and $\mathrm{U}$ as well as the fission studies, we will employ two new PhD students in the autumn of this year.

\section{Acknowledgements}

We wish to thank the technical staff of the The Svedberg Laboratory for skillful and enthusiastic assistance. The work is supported by the Swedish Nuclear Fuel and Waste Management Company, Swedish Nuclear Power Inspectorate, Ringhals AB, Forsmarks Kraftgrupp AB, Swedish Defence Research Agency, Swedish Nuclear Safety and Training Centre and the European Union.

\section{References}

[1] S. Dangtip, et al., A facility for measurements of nuclear cross sections for fast neutron cancer therapy, Nucl. Instrum. Methods Phys. Res. A 452 (2000) 484.

[2] U. Tippawan, et al., Light-ion production in the interaction of $96 \mathrm{MeV}$ neutrons with silicon, Phys. Rev. C 69 (2004) 064609 and Phys. Rev. C 73 (2006) 039902(E).

[3] U. Tippawan, et al., Light-ion production in the interaction of $96 \mathrm{MeV}$ neutrons with oxygen, Phys. Rev. C 73 (2006) 034611 [nucl-ex/0501014].

[4] V. Blideanu, et al., Nucleon-induced reactions at intermediate energies: New data at $96 \mathrm{MeV}$ and theoretical status, Phys. Rev. C 70 (2004) 014607.

[5] A. Prokofiev, et al., A new facility for high-energy neutron-induced fission studies, Proceedings of the International Conference on Nuclear Data for Science and Industry, AIP Conference Proceedings No. 769 (Melville, New York, 2005), 800.

[6] P. Mermod, et al., Search for three-body force effects in neutron-deuteron scattering at $95 \mathrm{MeV}$, Phys. Lett. B 597 (2004) 243.

[7] P. Mermod, et al., $95 \mathrm{MeV}$ neutron scattering on hydrogen, deuterium, carbon and oxygen, submitted to Phys. Rev. C.

[8] H. Condé, et al., A facility for studies of neutron induced reactions in the $50-200 \mathrm{MeV}$ range, Nucl. Instrum. Methods Phys. Res. A 292 (1990) 121.

J. Klug, et al., SCANDAL - a facility for elastic neutron scattering studies in the $50-130 \mathrm{MeV}$ range, Nucl. Instrum. Methods Phys. Res. A 489 (2002) 282.

[9] S. Pomp, et al., The new Uppsala neutron beam facility, Proceedings of the International Conference on Nuclear Data for Science and Industry, AIP Conference Proceedings No. 769 (Melville, New York, 2005), 780.

[10] A. Prokofiev, et al., A new neutron beam facility at TSL, these proceedings.

[11]D.T. Bartlett, et al., The cosmic radiation exposure of aircraft crew, Radiat. Res. Congress Proceedings 2 (2000) 719.

[12] T.W. Armstrong and B.L. Colborn, Predictions of secondary neutrons and their importance to radiation effects inside the international space station, Radiat Meas. 33 (2001) 229.

[13] M. Tubiana, J. Dutreix, and A. Wambersie, Introduction to Radiobiology, Taylor \& Francis, 1990.

[14] J. Blomgren and N. Olsson, Beyond kerma - neutron data for biomedical applications, Radiat. Prot. Dosim. 103 (2003) 293.

[15] Single-Event Upsets in Microelectronics, topical issue, edited by H.H.K. Tang and N. Olsson [Mater. Res. Soc. Bull. 28 (2003)].

[16] J. Blomgren, Bo Granborn, T. Granlund, N. Olsson, Relations Between Basic Nuclear Data and Single-Event Upsets Phenomena, p. 121 in [15]. 
[17] K. Johansson, et al., Energy-resolved neutron SEU measurements from 22 to $160 \mathrm{MeV}$, IEEE transactions on Nuclear Science I 45 (1998) 2519.

[18] K. Johansson, et al., Neutron induced single-word multiple-bit upset in SRAM, IEEE transactions on Nuclear Science I 46 (1999) 1427.

[19] M. Olmos, A. Prokofiev, and R. Gaillard, Unfolding procedure for SER measurements using quasi-monoenergetic neutrons, International Reliability Physics Symposium (IRPS 2005), San Jose, California, April 17-21, 2005.

[20] J.-C. Bourselier, TALYS Calculations for Evaluation of Neutron-Induced Single-Event Upset Cross Sections, UU-NF 05\#07, ISSN 1401-6269.

[21] J. Blomgren, Experimental activities at high energies, invited lecture at Workshop on Nuclear Data for Science \& Technology: Accelerator Driven Waste Incineration, Trieste, Italy, Sept. 1021, 2001.

[22] A. Carlson, et al., Update to nuclear data standards for nuclear measurements, Proc. Int. Conf. on Nuclear Data for Science and Technology, Trieste, Italy, May 19-24, 1997, Part II, 1223.

[23] P. Lisowski, et al., U, OECD/NEA Report NEANDC-305, 177. 